\title{
Dark Matter: The Nature of Space and the Galactic Gravitational Fields
}

\author{
Jacob Schaf \\ Instituto de Física, Universidade Federal do Rio Grande do Sul (UFRGS), Av. Bento GonÃ ğalves, 9500, CEP: 91501-970, Porto Alegre-RS, Brazil
}

Copyright (C) 2015 by authors, all rights reserved. Authors agree that this article remains permanently

open access under the terms of the Creative Commons Attribution License 4.0 International License

\begin{abstract}
The non-Keplerian rotation of the galaxies is actually among the most challenging troubles of the current theories with space and gravitation. The aim of this work is showing that the origin of this impasse is a wrong view about the nature of space and the gravitational physics. The Higgs theory introduces the real Higgs Quantum Space (HQS), giving mass to the elementary particles. This HQS thus rules the inertial motion of matter and propagates light. It is locally the ultimate reference for rest and for motions and thus directly involved in the gravitational physics. Recent experimental observations, achieved with the help of the tightly synchronized atomic clocks in orbit, demonstrate that such a real HQS effectively exists and is circulating round earth and the sun according to a Keplerian velocity field, consistent with the planetary motions. This directly explains the null results of the light anisotropy experiments and accurately creates the observed gravitational dynamics on earth and in the solar system. Specifically the form of the galactic velocity field, created by the orbiting stars, is shown to deviate considerably from the Keplerian form, which explains the observed non-Keplerian rotation of the galaxies without the need of dark matter.
\end{abstract}

Keywords Dark Matter, Higgs Quantum Space, Gravitational Physics, Keplerian Velocity Field

\section{Introduction}

The distribution of the visible matter within galaxies is rather different from that of a compact astronomical body like our sun. Almost the whole mass of a galaxy (more than 99\%) is orbiting in the form of stars round the galactic nucleus under the collective self-consistent gravitational field, created by them. In strong contrast, more than $99 \%$ of the mass of the solar system is concentrated in the central static sun.

Galaxies have a central bulge, formed by elliptically and non-equatorially orbiting stars, which normally harbors in the center a super-massive black hole. Beyond the bulge, up from about $10^{4}$ light years, galaxies are composed of a swarm of stars, orbiting within a thin disk along nearly circular orbits round the galactic center that extends out to about $5 \times 10^{4}$ light years. The stars are separated from each other by large distances, in the order of light years, so that most of the space within a galaxy is empty. Our Milky Way Galaxy is an old barred spiral galaxy having a central bulge of about $10^{4}$ light years across, where it harbors a super-massive black hole. The bulge is surrounded by a disk-shaped swarm of hundreds of billions of stars, orbiting along closely circular orbits, extending out to about $4.5 \times 10^{4}$ light years.

Many published works [1, 2, 3] display orbital velocity profiles, obtained by spectroscopic methods, for the stars in galaxies as a function of the distance from the galactic center. Velocity profiles of the Andromeda galaxy, of our Milky-Way galaxy as well as of a number of other galaxies are available in the literature. They all show that the orbital velocity of the stars falls not off with distance as predicted by the current theories of gravity. In many galaxies, the orbital velocity even increases with distance. Fig.1 displays an observed velocity profile of our Milky Way Galaxy together with a typical Keplerian rotation curve. From the viewpoint of the current theories, the rotation rate of the galactic disks are much too fast to be accounted for by the gravitational force or spacetime curvature, produced by their content and distribution of visible matter.

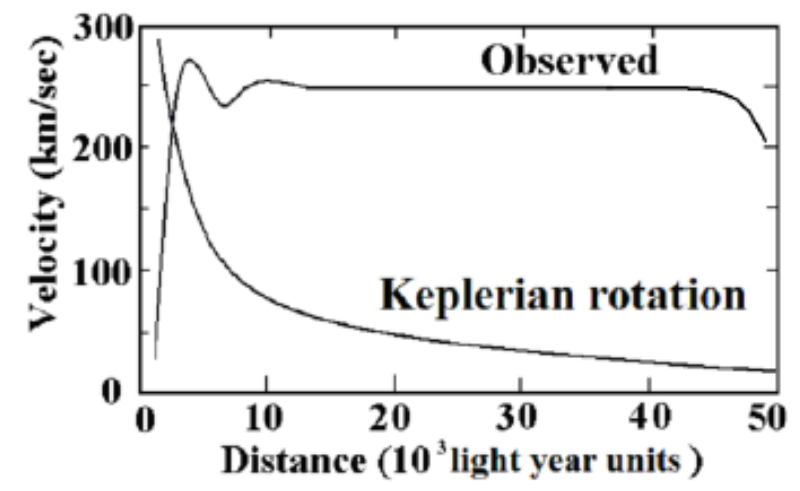

Figure 1. An observed rotation profile of the Milky Way galaxy as a function of the distance from the galactic center, together with the typical Keplerian rotation curve, from Ref. [3].

These discrepancies between theory and observations, instead of having raised suspicions about the current theories of space and gravitation, have lead to distrusts on the observations of the astronomers. It is alleged that the observational means visualize only a small part of the matter content of the 
galaxies and that a huge amount of invisible gravitating (dark matter) is present, causing the non-Keplerian rotation. Challengingly however, the amount of such dark matter, necessary to account for the observed galactic rotation rate, is estimated to be about 5 times larger than that of the total visible matter. This in fact must be seen as a measure of the wrongness of the current view about the nature of space and the gravitational physics. To present date nobody has idea where such a huge amount of dark matter could be hided and what its nature is. In fact, the nature of dark matter must be exceedingly exotic. It interacts with ordinary matter only by gravity and does not scatter, absorb or emit electromagnetic radiation. Actually, a variety of very massive hypothetical non-hadronic particles (MACHOS, WIMPS etc.) is being proposed as possible candidates for dark matter. Some people assert that an external halo of dark matter is responsible for the non-Keplerian galactic rotation. However, if dark matter interacts with ordinary matter by gravity, why then is it not concentrated within stars and within the galaxies? The non-Keplerian galactic rotation is actually among the most challenging impasses in the current theories of space and gravitation. Another way to address the problem of the galactic rotation rate, is by the gravitational potential. Several authors have computed the gravitational potential $[U(r)]$ as a function of the distance from the galactic nucleus for our Milky Way galaxy as well as for other galaxies, with base in the current theories of gravitation, taking into account the observed matter density in these galaxies. See for instance reference [4]. Systematically all these computed gravitational potentials are inconsistent with the observed orbital motions of the stars in the galactic disk.

The gravitational potential can of course be calculated by an empirical method too, using the circular orbital velocity profile of the stars, like that depicted in Fig.1. According to the Virial Theorem, the gravitational potential $U(r)=-(\gamma M / r)$ has a very simple relation with the circular orbital velocity $V_{\text {orb }}(r)$ in a central gravitational force field:

$$
U(r)=-V_{\text {orb }}^{2}(r)
$$

Fig.2 displays the gravitational potential $U(r)$, calculated with the help of Eq.(1), using the observed velocity profile in Fig.1. Please see especially the region below the external galactic border. The galactic gravitational potential curve, obtained in this way, is to be seen as the true observed potential. The result shows that this gravitational potential is closely leveled within the galactic disk, which means that the gravitational acceleration $a=-\operatorname{grad} U$ toward the galactic center is almost zero (no central force). Hence, in spite of the absence of a central force, the stars move along circular orbits. In his General Theory of Relativity (GR) Einstein tried to explain the gravitational dynamics in terms of spacetime geometry. Nevertheless, GR too cannot explain the observed galactic gravitational dynamics and actually GR moreover is in conflict with several clear-cut experimental observations, as will be shown in the next section.

The unsolvable impasse of the current gravitational theories with the galactic gravitational dynamics arises mainly from the fact that within the galactic disk the stars move along circular orbits in the absence of a central force field. The fact that the orbital velocity of these stars does not fall with distance, as predicted by these theories has no explanation to present date. In some galaxies, the orbital velocity of the stars even increases with distance from the galactic center. None of the current theories of gravity, including GR, can ex-

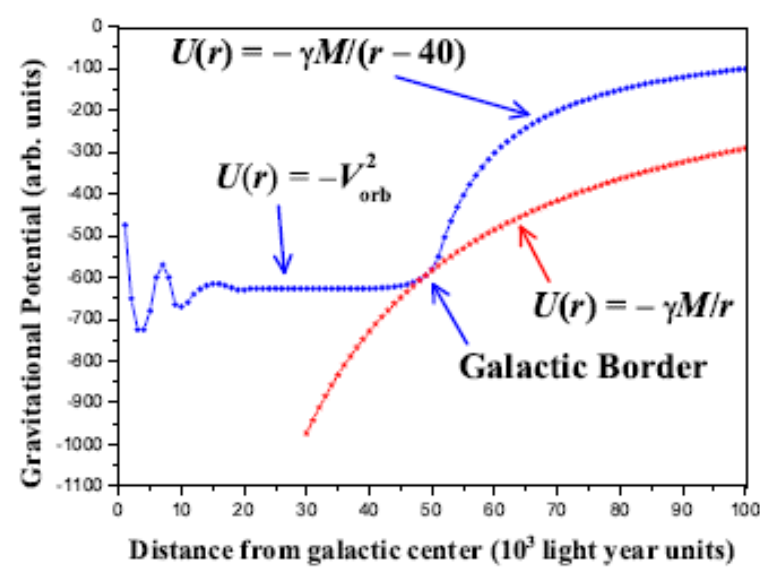

Figure 2. The figure depicts the gravitational potential, obtained, below the galactic border, making use of Eq.(1) and the observed orbital velocities in Fig.1. This is to be seen as the observed gravitational potential curve. Beyond the galactic border the potential is assumed to follow closely the usual $(1 / r)$ dependence, where however $r$ is the distance on from the galactic border. This figure also displays a typical gravitational potentail curve $U(r)=-\gamma M / r$, normalized to the observed value of $U$ at the galactic border, where however $r$ is the distance on from the galactic center.

plain this galactic gravitational dynamics without postulating a huge amount of dark matter. In reality, the trouble with the galactic gravitational dynamics is not an isolated difficulty of these current theories of gravity. Actually, a series of clearcut experimental observations, achieved with the help of the tightly synchronized atomic clocks in orbit, appoint other difficulties, some of which are even much more deleterious than the impasse with the galactic rotation. This lets clear that the only possible way to solve the impasses with gravity and the galactic gravitational dynamics is disclosing the true nature of the gravitational fields.

\section{The Nature of Space and of the Gravitational Fields}

With the help of the atomic clocks, orbiting in satellites round earth and collectively synchronized to within $0.1 \mathrm{~ns}$, the one-way velocity of electromagnetic (EM) signals (light) between satellites has been precisely measured. Especially clear-cut measurements were achieved between the robotic twin satellites of the Gravity Recovery and Climate Experiment (GRACE). [5] These twin satellites move in the same sense at nearly $8 \mathrm{~km} / \mathrm{sec}$ along coplanar and practically identical circular polar orbits at about $500 \mathrm{~km}$ of altitude, separated from each other by closely $200 \mathrm{~km}$ and their positions being monitored by the GPS within $3 \mathrm{~cm}$. These twin satellites constitute a well-defined inertial reference.

It has been observed that the signal transit time from the leading satellite to the rear satellite corresponds to a shortening by more than five meters (17ns) with respect to the expectations of the Special Theory of Relativity (STR). However, the signal transit time from the rear satellite to the leading satellite is lengthened by more than five meters $(17 n s)$. These discrepancies correspond in both cases exactly to the distance moved by the receiving satellite during the signal transit time and are consistent with backward signal anisotropy of nearly $8 \mathrm{~km} / \mathrm{sec}$, which is exactly the velocity of the satellites. This anisotropy is two orders of magnitude larger than the experimental precision of the experiment and shows that 
the EM signal (light) has a well-defined and isotropic NorthSouth velocity $c$ within the geostatic non-rotating reference, the same with respect to which the satellites are moving at 8 $\mathrm{km} / \mathrm{sec}$.

This measured one-way anisotropy of light unambiguously proves, by the first time, that a spatial medium (possibly the Higgs Quantum Space (HQS)) exists, which propagates light at the characteristic constant velocity $c$ with respect to this medium and not with respect to Einstein's inertial references. This result by it alone invalidates the fundamental assumptions of the Special Theory of Relativity, according to which the velocity of light is intrinsically constant and isotropic with respect to all possible inertial references. It also shows that this medium propagating light is not moving along the North-South direction with respect to Earth. This one-way anisotropy is due exclusively to the motion of the satellites. This observation conclusively breaks by the first time the century old believe that the velocity of light is intrinsically constant and isotropic with respect to all possible inertial references.

The immediate consequence of this observation is the absolute need of a new interpretation of the light anisotropy experiments, performed in the past century. Most of the Michelson experiments measured the light anisotropy, due to the orbital and cosmic motion of earth. Systematically, all these experiments obtained nominally null results. Now this must be interpreted as proving that earth has no resultant velocity with respect to the HQS propagating light. Obviously this can make a sense only if the HQS moves with earth round the sun and with the solar system round the galactic center etc. Hence, the observed isotropy of light with respect to earth does not corroborate Einstein's postulate of the intrinsic isotropy of light, however simply reveals the true kinematical circumstance of earth with respect to the HQS propagating light. The experimental observation, to be described in the subsequent paragraphs, confirms and deepens these conclusions.

In his GR, Einstein denies the existence of the Newtonian gravitational forces, because of the fact that they cannot be real. He explains the orbital motions within gravitational fields in terms of curved geometry of spacetime. In differential form, the metric of the curved spacetime, near to a spherically symmetric mass $M$ (Schwarzschild), is characterized by the invariant length of the four-dimensional line element $d s$. In terms of spatial spherical coordinates and time, the invariant $d s$ is given by:

$$
d s^{2}=\left[1-\frac{2 U}{c^{2}}\right]^{-1} d r^{2}+r^{2} d w^{2}-c^{2}\left[1-\frac{2 U}{c^{2}}\right] d t^{2}
$$

where $U=\gamma M / r$ is the value of the gravitational potential as a function of the radial coordinate $r, \gamma$ is the gravitational constant, $d w$ is the angle subtended by $d s$, and $c$ is the velocity of light, as measured by the go-return light roundtrip and clock method. In GR, the motion of bodies along curved paths, within the gravitational fields, is viewed as generalized inertial motion along geodesic lines in the appropriately curved spacetime. This spacetime curvature is introduced to explain the curved orbital motions without the need of a central field of Newtonian gravitational forces.

Clearly, in this spacetime curvature, the gravitational time dilation in the last term in Eq.(2) plays a fundamental role. It predicts the slowing of the GPS clocks by the solar gravitational potential $U(r)$, according to $T=T_{0}\left(1-2 U / c^{2}\right)^{-1 / 2}$, where $T_{0}$ is the time rate of the clocks when $U=0$. To first order, the predicted slowing of the GPS clocks is proportional to $U / c^{2}$.

According to GR, the effect of the solar gravitational potential on the GPS clocks, having orbital plane closely parallel to the earth-sun axis, during the 6 hours closer (than earth) from the sun, should cause a total delay of more than $24 \mathrm{~ns}$, which would be recovered during the 6 hours farther from the sun. The corresponding 12 hours periodic sinusoidal variation in the time display of the GPS clocks with respect to the reference clock on earth, would be more than two orders of magnitude larger than the stability and precision of these clocks within this period. As the gravitational potential is a scalar, the gravitational slowing of a stationary or a moving clock within the gravitational field must be exactly the same.

Observation nevertheless shows that, while the gravitational slowing of clocks, stationary with respect to the ordinary space coordinates within gravitational fields (on earth), agrees with the prediction of GR, the GPS clocks, moving with earth round the sun, show no sign of the gravitational slowing, due to the solar gravitational field.[6, 7] GR cannot explain this absence because the gravitational potential is a scalar. This observational fact has very drastic consequences on the credibility of GR. It demonstrates that the slowing of clock rate (time dilation) is not due to the gravitational potential. On the other hand, the observed slowing of clocks within the earth-based laboratories cannot be due to relative velocity, because these clocks rest with respect to the laboratory observer. Time dilation is well-observed for particles at very high velocity with respect to the terrestrial laboratories. If the slowing of clocks (time dilation) is not due to the gravitational potential and not to relative velocity too, then it must be due to another velocity of a more fundamental nature, which clearly is the velocity with respect to the local HQS. The absence of the gravitational time dilation on the GPS clocks fully corroborates the above conclusions from the one-way anisotropy of light, measured with the help of the GRACE twin satellites, according to which earth is stationary with respect to the HQS. These facts inexorably cause breakdown of the theory of GR. They demonstrate that the explanation of gravity in terms of spacetime geometry, despite more precise than Newtonian gravity, is fictitious as much as the explanation in terms of the Newtonian gravitational forces. Spacetime curvature is not a physical explanation of gravity, but simply mimics the observations.

Some people [8] claim that the gravitational slowing of the GPS clocks, due to the solar field, is absent, because the GPS satellites, together with earth, are free falling in the solar gravitational field and hence are not accelerated. However, from this viewpoint, the same GPS clocks are also free falling in the earth's field and notwithstanding undergo exactly the gravitational slowing predicted by GR. However, please see the true reason for this in Section VII-9 of Reference [9] Note that in the view of GR, the local inertial references in a gravitational field are free-falling and hence, clocks stationary within earth based laboratories are accelerated upward. According to the above allegations, the gravitational slowing of clocks resting on earth is due to this upward acceleration. However, experiments with Muons in cyclotrons have shown that accelerations up to $10^{19} \mathrm{~m} / \mathrm{sec}^{2}$ cause no effect on the mean life time of the Muons.[10] This observation definitively refutes the idea that acceleration causes slowing of clocks (time dilation). 
Einstein introduced the spacetime curvature to explain the circular orbital motions within the gravitational fields without a central force field. However, the absence of the gravitational time dilation, due to the solar gravitational field, on the GPS clocks, moving with earth round the sun, puts in check this central idea of GR. If the solar gravitational time dilation is absent in the orbiting earth, obviously the lengthening of the radial coordinate in the first term of Eq.(2) must be absent too. Otherwise the length o $d s$ cannot be constant. This cancels the spacetime curvature due to the solar mass. If the Newtonian gravitational forces cannot be real and if on the orbiting planets the spacetime curvature, caused by the sun is absent, earth and the other planets of the solar system are moving round the sun along circular orbits in flat spacetime and in the absence of a central force field. This breaks fundamental principles of physics. In a static situation, the spacetime curvature of GR could even be helpful to explain the gravitational pull. It however fails completely in the dynamic situation. The inexorable conclusion is that the planets move along circular orbits round the sun, not because of central Newtonian gravitational forces and also not because of spacetime curvature. They move so because of another reason that will be disclosed hereafter in terms of Higgs Quantum Space dynamics.

An appropriate theory of gravity must of course be able to explain consistently the gravitational dynamics on earth and in the solar system as well as the non-Keplerian rotation of the galaxies. It moreover must be able to explain all the effects of the gravitational fields on the propagation of light and on the rate of the clocks in terms of true physical effects. The recently published new theory of gravitation, [9] is exactly such a theory. Please see also references [11, 12, 13]

The superconducting condensate (SCC) is well-known to confine the electromagnetic (EM) field. It confines the electric field by displacement of the Cooper electron pairs and the magnetic field by developing a quantized velocity field (screening currents) of the SCC round the magnetic field. This is the Meissner effect, providing inertial mass to the photons within the superconductor. [14] This momentous achievement was an essential clue to disclose the origin of the inertial mass of the elementary particles. According to the Higgs theory, a scalar Higgs field or condensate of Higgs bosons (HC), analogous to the SCC, fills up the whole of space. Analogously as the SCC confines the EM field, this $\mathrm{HC}$ confines the weak nuclear field and indirectly all the elementary particles porting hypercharges, thereby providing them with inertial mass. This is the Higgs mechanism, $[15,16]$ the analog of the Meissner effect in superconductivity. It is responsible for the mechanical properties of the elementary particles. This lets clear that the Higgs Quantum Space (HQS) rules the inertial motion of matter and the propagation light and hence is locally the ultimate (locally absolute) reference for rest and for motions.

To say that the HQS is the locally absolute reference for rest and for motions of matter and light has a very profound meaning. It means that, if the HQS is locally moving, according to a certain velocity field $\vec{V}(r, \theta, \phi)$, with respect to the ordinary space coordinates $(r, \theta, \phi)$, the local absolute reference is moving accordingly. It also means that a particle locally commoving with the circulating HQS, corresponds locally to absolute rest. However, a particle stationary with respect to the ordinary space coordinates at a fixed point $(r, \theta, \phi)$ within the velocity field of the HQS, will be mov- ing (implicitly) with an absolute velocity $-\vec{V}$. This velocity is implicit (imaginary), because it cannot be described with respect to the ordinary space coordinates. Yet, this implicit velocity has real and tangible physical consequences that we continuously observe on earth. They are the gravitational acceleration or the gravitational pull and the circular or elliptic orbital motions within the gravitational fields.

In the presence of a magnetic field, a superconductor develops a macroscopic velocity field of the SCC (macroscopic screening currents) and a Lorentz macroscopic force field, expelling the magnetic field out from the superconductor or compressing it into regions, where the superconducting order parameter is weaker. This way the SCC reduces its energy within the Bose-Einstein correlation potential well. This is a macroscopic manifestation of the Meissner effect. The presence of the matter field of a matter body introduces phase disorder, depressing locally the Higgs order parameter and elevating the energy of the HC. The HQS can lower its energy by developing a macroscopic velocity field $\vec{V}(r)$ (screening currents) round this body and an inertial force field (fictitious gravitational force field), thrusting the matter fields toward this body. This is a macroscopic manifestation of the Higgs mechanism. The observations, described above, show that this velocity field is proportional to $(M / r)^{1 / 2}$, where $M$ is the source mass and $r$ is the spherical radial coordinate. For a spherically symmetric body, the velocity field of the HQS round the body has the form of a spherically symmetric Keplerian velocity field, consistent with the local main astronomical motions:

$$
\vec{V}(r)=[\gamma M / r]^{1 / 2} \vec{e}_{\phi}
$$

In this equation, $\gamma$ is the Newtonian gravitational constant and $\vec{e}_{\phi}$ is the unit vector along the azimuthal spherical coordinate.[9] This velocity field is a cylindrical velocity field consistent with the local main astronomical motions, where the magnitude of the velocity for a given $r$ has the same value for all $\theta$ and all $\phi$. This leads to the spherical symmetry of the gravitational field. In the Keplerian velocity field round the sun, the planets are stationary with respect to the moving HQS. Such a Keplerian velocity field of the HQS, is assumed to be present round each astronomical body (galaxies, stars, planets or asteroids) throughout the universe.

According to the new theory of space and gravitation in Reference [9], experimental observations show that the velocity of light has a well-defined value $c_{H Q S}$ with respect to the local HQS and not with respect to all the possible inertial references. The velocity $v_{H Q S}$ of the elementary particles with respect to the HQS (and not the relative velocity), together with this velocity of light $c_{H Q S}$ (and not the velocity of light measured by the light go-return roundtrip and clock method), rule all the so called relativistic effects on time (slowing of clocks), on mass (relativistic mass) and on energy (relativistic energy) of the elementary particles.[17]

From the viewpoint of this new theory, the orbital motion of the astronomical bodies, throughout the universe, is mostly motion of the HQS itself in the Keplerian velocity fields, creating the respective gravitational fields. The main natural astronomical bodies, like planetary satellites, the planets of the solar system and the stars in the galaxies are all commoving with the HQS along nearly circular orbits, closely within the equatorial plane of the respective velocity field of the HQS. This minimizes their velocity with respect to the HQS and is the reason why these systems are disk shaped. The planetary satellites, the planets of the solar system and the stars in the 
galactic disk almost truly rest with respect to the HQS. This explains the null results of the Michelson light anisotropy experiments, searching for light anisotropy, due to the orbital and cosmic motion of earth. It also explains the observed absence of the gravitational slowing of the GPS clocks and all clocks moving with earth round the sun, due to the solar field. For details of the reason why the GPS clocks, orbiting at 55 degrees with respect to the earth's equator, are slowed by the earth's field, please see Section VII-9 of Reference [9]. Unfortunately, Einstein and physicists along a whole century have not perceived that the null results of the Michelson light anisotropy experiments are exactly the signature of the physical mechanism of gravity in action. Another signature of this same physical mechanism of gravity is the absence of the gravitational slowing of all clocks, moving with earth round the sun, due to the solar field.

Due to the increase of the $\phi$ velocity of the HQS with decreasing radial coordinate, characteristic of the Keplerian velocity field, the velocity distribution, through a small region in the $(r, \theta)$ plane round any given point of the ordinary space, corresponds to rotation of the HQS round a well-defined overhead axis. In fact, to each point in ordinary space, having different $r$ coordinates, there corresponds a different rotation axis and a different rotation rate. Hence, to a particle whose de Broglie wave fronts lie in the $(r, \theta)$ plane (velocity along $\phi)$, the local inertial reference (IR), valid at its position, is rotating at the same rate round the same overhead axis. In conventional physics, a rotating reference cannot be an inertial reference. However, here it is itself the space ruling the inertial motion of matter that is rotating. A matter particle stationary in the ordinary space, necessarily is implicitly moving within the respective local rotating IR, oppositely along a circular path, round the same overhead axis, under an upward centripetal force. This opposite circular motion of bodies is implicit, because it cannot be described with respect to the ordinary space coordinates. It is due to motion and rotation of the HQS itself in the ordinary space, which however generates real physical effects. As soon as the upward centripetal force is cut off, the body becomes free and will move instantaneously along an inertial path by rotating its velocity vector together with the local rotating IR. The gravitational acceleration of free bodies in this HQS-dynamics is simply the consequence of their instantaneous inertial motion within each local rotating IR. Please see Section VI of Ref. [9] for all the necessary details. In particular, the gravitational slowing of clocks, resting with respect to the ordinary space coordinates within a gravitational field, is not due to the gravitational potential, nor to relative velocity with respect to an observer. It is due to the motion of the HQS through the stationary clocks, or the implicit velocity of the stationary clocks with respect to the moving HQS.

From the viewpoint of this new theory of space and gravitation (HQS-dynamics), [9] the orbiting planets of the solar system are simply carried round the sun by the Keplerian velocity field of the HQS, creating the solar gravitational field. Therefore the circular equatorial orbital motion of the planets round the sun needs not to be explained anymore, because it is the HQS, the ultimate reference for rest and for motion that is itself so moving. Only the ellipticity and the non-equatorial orbital motions need an explanation. Similarly, the solar system and the other stars in the galaxy are carried round the galactic nucleus by the velocity field of the HQS, creating the galactic gravitational field. From the view- point of this new theory, the circular equatorial orbital motion of the planets in the solar system and the circular equatorial orbital motion of the stars in the galactic disk involve fundamentally no (absolute) motion at all and no acceleration with respect to the HQS. Therefore, they need no any physical action (force) to move so. Locally, these bodies remain closely stationary at the same (absolute) place, however the place is itself circulating along circular orbits. From the view of this new theory, the orbital motion of the planets and the nonKeplerian orbital motion of the stars within the galactic disk, despite the absence of a central field of Newtonian gravitational forces as well as the absence of spacetime curvature, violates no any principle of physics. These bodies rest and or move (slowly) rigorously according to the principle of inertia (wave propagation), with respect to the moving HQS.

\section{The Gravitational Dynamics of a Bi- nary Star System Unveils a Key Fea- ture Leading to the Non-Keplerian Galactic Rotation}

In the previous Section II it has been shown that, according to the new theory of space and gravity, [9] the circular orbital motions of the planets in the solar system and also the circular orbital motions of the stars in the galactic disk are very closely motion of the HQS itself. These bodies are very closely stationary with respect to the local HQS in the velocity fields of the HQS, creating the respective gravitational fields. This is the reason why the velocity of light is isotropic with respect to these bodies and also is the reason why the atomic clocks, moving with these bodies, run not slow, as shown by the GPS clocks that move with earth round the sun. [6, 7] However, why do the orbital velocities of the planets round the sun decrease according to the law of Kepler (Eq.(3)), while the orbital velocities of the stars in the galactic disk do not? The purpose of this section is showing that the new theory of gravity [9] predicts even with details the nonKeplerian galactic gravitational dynamics without the need of dark matter.

In the view of the current gravitational theories, effects of motion of the gravitational sources on their gravitational fields become relevant only for velocities close to the velocity of light. However, why is the velocity of the Moon, from the viewpoint of the solar non-rotating reference, so irregular? From the viewpoint of an observer in this solar static reference, the Moon moves considerably faster outside the earth orbit than inside. This in particular may be explained in terms of the equivalence of the inertial references (Galilean relativity). According to this equivalence, the Moon moves (uniformly) along a circular orbit round earth, in the non-rotating inertial reference attached to earth, under the fictitious Newtonian gravitational force between earth and the Moon. In its turn, the earth-Moon system moves (uniformly) in the solar non-rotating inertial reference, under the gravitational force between earth and the sun. However, the explanation in terms of the Newtonian gravitational forces is well-known to be fictitious, because these forces cannot be real. They have been postulated by Newton because of lack of a true physical explanation for gravitation. Einstein has rejected the Newtonian theory of gravitation because of this non-realness and explained the gravitational dynamics in terms of generalized 
inertial motions along geodesics in curved four-dimensional spacetime. However, GR cannot explain the observed galactic gravitational dynamics and now is in frontal conflict with several recent clear-cut experimental observations (please see the previous Section II).

From the viewpoint of the new theory of gravitation, [9] earth is locally stationary with respect to the HQS in the Keplerian velocity field, creating the solar gravitational field (Eq.(3)). This is essential to allow for the spherically symmetry (excepting only for small tides) of the earth's Keplerian velocity field, creating the earth's gravitational field. In its turn, the Moon is locally stationary with respect to the HQS in the earth's Keplerian velocity field. Hence, the regular motion of the Moon in the earth-Moon system is much more than simply a case of Galilean relativity. While earth is resting with respect to the HQS in the solar Keplerian velocity field, the Moon is resting in the earth's Keplerian velocity field. Both bodies are locally in very close absolute rest with respect to the HQS, the ultimate reference for rest and for motion. The observed relative motions of these bodies, as viewed by an observer in the solar non-rotating reference, are, in a strictly physical sense, simply apparent motions. They are motions of space itself. In the new theory, it is important to distinguish between real motion of a particle with respect to the local HQS, which affects the internal structure of the particle, and the apparent motion, which is motion of the HQS itself in the ordinary space and which lets the particle, the velocity of light and the rate of clocks locally unaltered. For instance, despite the apparent (relative) motions between earth and Venus from the viewpoint of the solar non-rotating reference, they are both very closely stationary with respect to the local HQS in the solar Keplerian velocity field. Light anisotropy and atomic clock-rate experiments confirm that they effectively are stationary with respect to the HQS.

Here, it will be shown that, from the viewpoint of the inertial reference of a hypothetical static observer, the relative motion of a gravitational source can have important effects on the observed velocity field of the HQS, creating its gravitational field, even at low velocities. Such effects are obviously very important in the galactic gravitational dynamics, as, from our viewpoint, almost the whole matter, creating the galactic gravitational field, is moving in the ordinary space round the galactic nucleus. Each orbiting star of course carries along the orbit its Keplerian velocity field of the HQS (Eq.(3)) and therefore, taking such effects into account is essential to produce the non-Keplerian galactic rotation.

To build up the galactic velocity (gravitational) field, it is necessary that the velocity fields, creating the gravitational fields of the individual stars, be fairly well polarized. In the solar system the planets and their satellites rotate and orbit almost all in the same sense along nearly circular equatorial orbits within the plane of the solar system. This demonstrates that the Keplerian velocity fields, creating the gravitational fields of all these bodies are well polarized and rotate in the same sense round axes nearly parallel to the axis of the solar system. In the galactic disk, the stars too are orbiting in the same sense along nearly circular equatorial orbits round the galactic center. However, presently no information about the orientation of their planetary orbits and velocity fields of the HQS, creating their gravitational fields can be established. Nevertheless, it seems reasonable to assume that the rotation axes of the star velocity fields of the HQS, creating the stellar gravitational fields, are fairly well polarized. They may be rotating dominantly in the same sense as the galaxy, round axes that make an angle lower than 90 degrees with the rotation axis of the galaxy. The rotation axis of our solar system is known to make about 63 degrees with respect to the rotation axis of the Milky Way galaxy. This angular deviation may be related with the spiraled structure of the galaxy. Adequate addition of the star velocity fields must generate the galactic velocity field of the HQS that rules the galactic gravitational dynamics.

In order to highlight the relevance of the effects of the orbital motion of the gravitational sources on the collective velocity field of a system of bodies gravitating in their selfconsistent field, let us begin with the simple case of a binary system. The gravitational dynamics of such a binary system discloses the effect of the orbital motion on the velocity fields creating their gravitational fields, which is the essential clue to understand the non-Keplerian galactic gravitational dynamics. Consider two bodies of masses $M_{1}=M_{2}$ $=M$, moving in the same circular orbit round their center of mass (CM) and the velocity fields, creating their gravitational fields, rotating in the same sense as the orbital motion about parallel axes, as shown in Fig.3. Note that this configuration of the velocity fields is the only one leading to a stable system. These stars will be commoving with the HQS along circular orbits in the equatorial plane of the collective velocity field of the HQS, creating the gravitational field of the binary, as observed in many binaries. It can be shown that stars with oppositely rotating velocity fields of the HQS cannot form a bound system. The gravitational dynamics of the binary system can be well described by Newtonian mechanics. Balance of the mutual Newtonian gravitational forces $\gamma M^{2} /\left(2 x_{0}\right)^{2}$ and of the centripetal force $M v_{o r b}^{2} / x_{0}$ on each star leads to the observed orbital velocity $v_{\text {orb }}$ of each star round the $\mathrm{CM}$ :

$$
\nu_{\text {orb }}=\frac{1}{\sqrt{2}}\left(\gamma M / 2 x_{0}\right)^{1 / 2}
$$

In this expression, $x_{0}$ is positively defined. Consider now a small test particle moving round an equal, however static, mass $M$ in a circular equatorial orbit having the same radius $2 x_{0}$. The orbital velocity $v_{\text {orb }}^{\prime}$ of this test particle would be considerably larger:

$$
\nu_{o r b}^{\prime}=\left(\gamma M / 2 x_{0}\right)^{1 / 2}=\sqrt{2} \nu_{o r b}
$$

From the viewpoint of the Newtonian gravitational theory there is obviously nothing wrong with Eqs.(4) and (5). However, although these equations match precisely the observations, the troubles begin as soon as it is remembered that the Newtonian gravitational forces cannot be real. The explanation in terms of the Newtonian gravitational forces is well-known to be fictitious. In his General Relativity (GR), Einstein has refuted the Newtonian theory of gravitation, because of this non-realness. He has proposed a new theory of gravitation that explains the gravitational dynamics in terms of generalized inertial motion along geodesics in the curved geometry of the four-dimensional spacetime. However, now GR too is in serious trouble. It cannot account for the nonKeplerian rotation of the galaxies and, what is even much more deleterious, is that it also cannot explain the absence of the gravitational slowing of the GPS clocks, due to the solar gravitational field. This gravitational time dilation is predicted by GR, but not observed. It moreover cannot explain 
the one-way light anisotropy, precisely measured with the help of the tightly synchronized atomic clocks in the GRACE twin-satellites (please see beginning of Section II)

However, from the viewpoint of the new theory of gravitation,[9] the Higgs Quantum Space (HQS), ruling the inertial motion of matter and the propagation of light, is moving according to a Keplerian velocity field round each astronomical body (please see Eq.(3)). According to this theory, the orbital motions of the planets and of the main natural astronomical bodies in general, throughout the universe are mainly motions of the HQS itself. These bodies are all closely stationary with respect to the HQS, which straightforwardly explains the null results of the Michelson light anisotropy experiments and also the absence of the gravitational slowing of the GPS clocks, due to the solar gravitational field. In reality, the circular equatorial orbital motion of the astronomical bodies within gravitational fields needs not to be explained anymore, because it is the HQS itself, the ultimate reference for rest and for motions that is so moving. On the other hand, all deviations from this circular equatorial motion, like the elliptical and the non-equatorial orbital motions or the free-fall on earth, are explained straightforwardly in terms of the inertial motion of these bodies with respect to the locally moving and warping HQS.[9] This theory also accounts, in terms of well-known and true physical effects, for all the observed effects caused by gravitational fields on the propagation of light and on the rate of clocks. Here it will be shown that this HQS-dynamics in addition predicts with details the observed non-Keplerian gravitational dynamics of galaxies, without the need of dark matter.

From the viewpoint of the HQS-dynamic gravitational mechanism, in the case of Eq. (5) the source of the HQS velocity field remains practically stationary at the CM. Therefore Eq.(5) gives the local velocity of the HQS in the Keplerian velocity field round a static mass $M$, exactly as stipulated by Eq.(3). However, in the dynamic case of the binary stars, Eq.(4) too gives the value of the velocity of the HQS, at the site of $M_{1}$ and at the site of $M_{2}$, due to the respective companion star. Otherwise, it would be impossible to their orbits to be circular. Hence, both Eqs.(4) and (5) describe the velocity of the HQS in the respective velocity fields. However, why are these velocities so different? The situation would of course be very different if the gravitational sources were not in orbit (initially static), in which case the velocity fields of $M_{1}$ as well as $M_{2}$ would be given by Eq.(3) and both masses would be moving with an implicit velocity (with respect to the HQS), given by the negative of Eq.(3). In this case however the system would be completely unstable and both sources would accelerate (fall) toward the CM, under the mutual influence on their local inertial references exactly as observed.

In the view of the present work, the difference between Eqs. (4) and (5) unveils a key feature that is at the origin of the non-Keplerian rotation of the galaxies. This difference discloses the effect of the orbital motion of the gravitational sources on the velocity field of the HQS, creating their respective gravitational fields, as viewed by an observer in the static non-rotating (XY) reference (please see Fig.3).

However, in the opinion of an observer, moving together with one or the other source of the binary, the source is stationary with respect to the local HQS and the velocity field of the HQS, creating the local gravitational field of the individual source, is spherically symmetric, as given by Eq.(3), excepting only for small tides. This he can confirm by light anisotropy experiments and by the rate of his atomic clocks. Nevertheless, from the viewpoint of an observer stationary in the static non-rotating (XY) coordinate axes (please see Fig.3), which constitute the reference with respect to which the collective velocity field of the binary must be described, the velocity fields of the individual stars are quite asymmetric. The value of the velocity of the HQS on the $\mathrm{X}$ axis, is much larger outside the orbit of the binary than inside the orbit. Moreover, the sense of the velocity of the HQS inside the orbit is opposite to the orbital motion as well as opposite to the outside velocity field. From the viewpoint of the observer in this static (XY) reference, the centripetal (gravitational) acceleration of a free-body, falling near to the outer surface of a star of the binary, is considerably larger than near to the inner side. Newton would have explained this saying that, while outside the gravitational forces of the two stars add up, while inside they subtract. However, according to the present theory, in both situations, the bodies simple are commoving with the local HQS, the velocity of which is spherically symmetric round the individual stars.

All these features of the present HQS-dynamic gravitational mechanism are fundamental in the creation of the nonKeplerian gravitational dynamics of galaxies. They in fact do it without the need of dark mater. The only possible reason for the reduced velocity of the HQS, inward the binary orbit and the increased velocity outside the binary, from the viewpoint of the static (XY) observer (please see Fig.3), is the orbital velocity of the stars round the center of mass (CM). From the viewpoint of the observer in the fixed (XY) reference, the orbital velocity of the sources, given by Eq.(4), subtracts from the spherically symmetric velocity fields (Eq.3) toward the inner side and adds to it toward the outer side, as depicted in Fig.3.

In order to put in evidence the full effects of motion of the gravitational sources on their velocity fields, consider now, in addition, a small test particle moving in the collective velocity field along a circular orbit within the orbital plane of the binary, however sufficiently far away in order to minimize the asymmetries of the binary field. The orbital velocity $v$ of such a test particle is of course given by:

$$
v(r)=\sqrt{\frac{\gamma 2 M}{r}}
$$

where $r$ is the distance on from the CM of the binary. For large $r$ Eq.(6) too corresponds to the velocity of the HQS, however in the collective velocity field of the binary:

$$
V_{\text {coll }}(r)=\sqrt{\frac{\gamma 2 M}{r}}
$$

In order to reconcile the addition of the velocity fields of $M_{1}$ and $M_{2}$ outside the binary with the velocity in the collective velocity field, given by Eq.(7), the same orbital velocity, which reduces the velocity fields toward the inner side, must enhance them outward the binary orbit. It must increase the velocity field $V_{1}$ toward the left-hand side of $M_{1}$ and $V_{2}$ toward the right-hand side of $M_{2}$. At distances $r$ much larger than $2 x_{0}$, addition of the velocity fields of $M_{1}$ and $M_{2}$ must reproduce the value, given by Eq.(7). Note however that addition of the velocity fields are due to the different sources $M_{1}$ and $M_{2}$. The addition of these velocities along the axis of $\mathrm{X}$, must satisfy the sum rule:

$$
V(r)=\sqrt{\left|V_{1}^{2}\left(r_{1}\right) \pm V_{2}^{2}\left(r_{2}\right)\right|}
$$




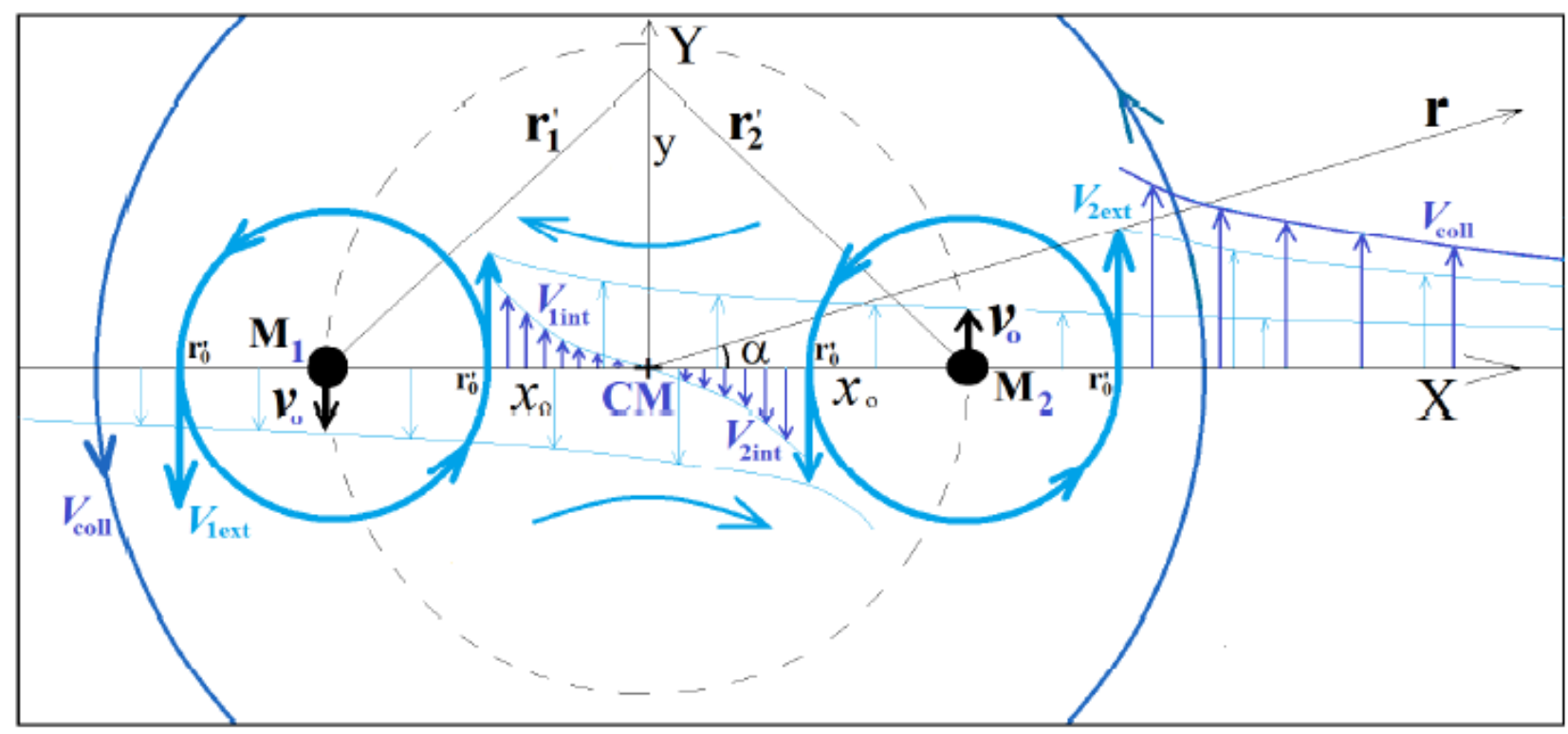

Figure 3. View of the velocity fields along the rotation axes of two masses $M_{1}=M_{2}=\mathrm{M}$ moving in the same sense in a same circular orbit round the center of mass $(\mathrm{CM})$ within the equatorial plane of the combined velocity field. The arrows, indicating the velocities are plotted to scale. Note that, from the perspective of the observer in the non-rotating [X,Y] coordinate axes, the velocity of the HQS, due to the velocity fields of $M_{1}$ and $M_{2}$ are larger outside than inside the binary orbit.

This sum-rule arises because of the fact that the velocity field of the HQS depends on the square-root of the source mass and distances. Note that the squares of $V_{1}$ and $V_{2}$ correspond to the gravitational potentials of respectively $M_{1}$ and $M_{2}$ as a function of $r$. Taking into account the sum rule Eq.(8), the addition of the upward velocity fields of $M_{1}$ and $M_{2}$, for large $x$ (large $r$ ) along the $+X$ axis in Fig.3, takes the form:

$$
V_{\text {coll }}^{2}=\frac{\gamma 2 M}{r}=\frac{1}{2} \frac{\gamma M_{1}}{x_{0}+x}+V_{e x t, 2}^{2}
$$

Note that $x_{0}$ is a positively defined distance. Solving for $V_{e x t, 2}$ along the $+X$ axis, for $x>x_{0}$, the velocity field of $M_{2}$ outside of the binary is given by:

$$
V_{e x t, 2} \sim \sqrt{\frac{3}{2}} \sqrt{\frac{\gamma M}{x-x_{0}}}
$$

A totally similar result is obtained to the left hand side of $M_{1}$, where $\left|V_{\text {ext }, 1}\right|=\left|V_{\text {ext }, 2}\right|=V_{\text {ext }}$.

Inserting the result of Eq;(10) into Eq.(9) and adding according to Eq.(8), the velocity field of the binary to the right hand side of $M_{2}$ (along $+X$ ), for large $x$, which means large $r$, is:

$$
V_{\text {coll }}=\sqrt{\frac{3}{2} \frac{\gamma M}{x-x_{0}}+\frac{1}{2} \frac{\gamma M}{x_{0}+x}} \sim(\gamma 2 M / r)^{1 / 2}
$$

This reproduces the result of Eq.(7) for large $r$. The procedure to obtain $V_{\text {coll }}$ to the left hand side of $M_{1}$ is analogous and the result is identical. These results show that effectively the same orbital velocity that, in the view of the static observer in the (XY) reference, reduces the velocity fields of $M_{1}$ and or $M_{2}$, given by Eq.(3) by a factor $(1 / 2)^{1 / 2}$ (see Eq.(4)) toward the inner side in Fig.3, enhances the velocity fields of $M_{1}$ and $M_{2}$ by a factor $(3 / 2)^{1 / 2}$ toward the outer side. This is of course not for nothing. Clearly, the reason is the orbital velocity of the sources in the static (XY) reference. This result shows that, while the contribution of $M_{1}$ to the collective velocity field, to the right hand side of $M_{2}$ on the axis of $\mathrm{X}$ is only $(1 / 4) V_{\text {coll }}$, the contribution of $M_{2}$ is
$3 / 4 V_{\text {coll }}$. An analogous conclusion is valid at the left hand side of $M_{1}$, however with the roles of $M_{1}$ and $M_{2}$ inverted. Hence, these effects of the orbital velocity on the velocity fields of the individual stars, do not affect the value of the collective (total) velocity field outside the binary. The collective velocity field, outside de binary, is the same as in the case the two sources are static.

In conclusion, inward the binary orbit $\left(|x|<\left|x_{0}\right|\right)$, along the axis of $\mathrm{X}$, the orbital velocity $v_{\text {orb }}$ of $M_{1}$ and or of $M_{2}$ subtract from the value of their respective spherically symmetric velocity fields (Eq.(3)), in consonance with Eq.(8):

$$
V_{i n t}=\sqrt{\frac{\gamma M}{r^{\prime}}-v_{o r b}^{2}}=\frac{1}{\sqrt{2}} \sqrt{\frac{\gamma M}{r^{\prime}}}
$$

where $r^{\prime}$ is the distance on from either $M_{1}$ or $M_{2}$ toward the inner side of the binary and beyond to respectively $+\infty$ or $-\infty$ along the $\mathrm{X}$ axis.

In the outer side $\left(|x|>\left|x_{0}\right|\right)$, the orbital velocity of $M_{1}$ and or of $M_{2}$ adds to their respective spherically symmetric velocity field Eq.(3), in consonance with Eq.(8):

$$
V_{e x t}=\sqrt{\frac{\gamma M}{r^{\prime}}+v_{o r b}^{2}}=\sqrt{\frac{3}{2}} \sqrt{\frac{\gamma M}{r^{\prime}}}
$$

where $r^{\prime}$ is the distance on from either $M_{1}$ or $M_{2}$, along the axis of $X$, toward the outer side of the binary and to $-\infty$ or $+\infty$.

The terms under the last square-root symbol in Eqs.(12) and (13) are identical to the gravitational potential of an isolated mass $M$. In the view of an observer, stationary on the $\mathrm{X}$ axis of the non-rotating (XY) reference, at the right hand side of $M_{2}$ and far away from the binary, $M_{2}$ contributes $(3 / 2) \gamma M / r$ to the total gravitational potential $U(r)=\gamma 2 M / r$ of the rotating binary and only $(1 / 2) \gamma M / r$ is due to $M_{1}$. An analogous conclusion is valid at the left hand side of $M_{1}$, where however the roles of $M_{1}$ and $M_{2}$ are inverted. The origin of the difference between the contributions of $M_{1}$ and $M_{2}$ to the effective gravitational potential is, besides their different positions, principally their orbital 
velocity. This view of the static (XY) observer is of fundamental importance, because it is exactly the view we usually make of the gravitational dynamics of the stars within galaxies. Note that in the view of the current gravitational theories, the gravitational potential depends only on the position of the sources. The effect of the low orbital velocities is totally irrelevant. However, outside the binary the total gravitational potential predicted by the two views are the same $U=\gamma 2 M / r$.

The results expressed by Eqs.(12) and (13) and plotted in Fig.3 are a unique feature of the present HQS-dynamic gravitational mechanism. It arises from the fact that the gravitational dynamics is created by the Keplerian velocity field of the HQS, which, for an isolated mass, is given by Eq.(3). None of the current theories of gravitation can give rise to the features expressed by Eqs.(12) and (13). These equations show that, while the velocity field, created by $M_{2}$ at its left hand side, is downward and has the value $-(1 / 2)^{1 / 2}\left(\gamma M / r^{\prime}\right)^{1 / 2}$, at the right hand side it is upward and has the value $+(3 / 2)^{1 / 2}\left(\gamma M / r^{\prime}\right)^{1 / 2}$. In these expressions $r^{\prime}$ is the distance to either sides on from the position of $M_{2}$. Hence, going in Fig.3 along the positive $\mathrm{X}$ axis, on from the $\mathrm{CM}$, there is an upward velocity step $\Delta V$, from $-(1 / 2)^{1 / 2}\left(\gamma M / r^{\prime}\right)^{1 / 2}$ to $+(3 / 2)^{1 / 2}\left(\gamma M / r^{\prime}\right)^{1 / 2}$, created by $M_{2}$ at a distance $r^{\prime}$ from it, the value of which is given by:

$$
\Delta V=\left[\sqrt{\frac{3}{2}}+\sqrt{\frac{1}{2}}\right] \sqrt{\frac{\gamma M}{r^{\prime}}} \sim 1.93 \sqrt{\frac{\gamma M}{r^{\prime}}}
$$

Note that this addition is not ruled by Eq.(8) because the velocities are of the same mass. Going along the $\mathrm{X}$ axis to the left hand side in Fig.3, on from the CM, there is an analogous velocity step at the position of $M_{1}$, however increasing the (opposite) downward velocity. Note that the magnitude of the velocity step depends on the mass $M$ as well as on $r^{\prime}$, the distance from this mass. In the case of the stars, orbiting in the galactic disk, this consideration must be made for each individual star. For instance, the velocity of the HQS in the solar Keplerian velocity field achieves $436 \mathrm{~km} / \mathrm{sec}$ at the solar surface. However, at distances of the first neighbor star (Alfa Centaury) the velocity is only about 60 meters $/ \mathrm{sec}$. The sun thus creates on its surface a velocity step of $872 \mathrm{~km} / \mathrm{sec}$ from side to side and only of about 120 meters/sec at the distances of Alfa Centaury. These velocity steps are a key feature that is fundamental in the building up of the velocity field of the HQS, ruling the non-Keplerian galactic gravitational dynamics. In this building up, it is important to perceive that the (upward) orbital velocity of $M_{2}$, given by Eq.(4), does not only increase the upward velocity at the right hand side of $M_{2}$, according to Eq.(13), however also decreases the downward velocity at its left hand side by the same amount, as given by Eqs.(12). This shows that the total magnitude of the velocity step, created by $M_{2}$, is the same as in the static case, however shifted by the orbital velocity.

The obvious conclusion from the above analysis is that the Keplerian velocity fields of the individual stars in the galactic disk act in the sense of opposing the Keplerian $(1 / r)^{1 / 2}$ decrease of the collective velocity field in the galactic velocity field. In this modification of the original Keplerian dependence $(1 / r)^{1 / 2}$ the velocity outside the orbit, increased by the orbital velocity as well as the velocity inside the orbit, decreased by the orbital velocity, both act together in the sense of reducing the Keplerian decrease of the velocity with distance from the galactic center. In some galaxies this effect is strong enough to invert the velocity gradient so that the velocity increases with $r$. However, beyond the border of the galactic disk, where the mass density falls strongly, the galactic velocity field of course regains the Keplerian $(1 / r)^{1 / 2}$ dependence.

In-between the orbiting stars of the binary system the velocity fields of $M_{1}$ and $M_{2}$ at the axis of $\mathrm{X}$, are opposite to each other and also both are opposite to the external velocity field (please see Fig.3). Moreover, they are reduced by their orbital velocity as shown by Eq.(4) and Eq.(12). Along the $\mathrm{X}$ axis, the addition of these internal velocity fields, according to Eq.(8), shows that, close to $M_{1}$, the upward velocity field of $M_{1}$ dominates and close to $M_{2}$ the downward velocity field of $M_{2}$ is dominant. In-between the two masses, the resultant collective velocity field, as a function of the position $x$ and along the axis of $\mathrm{X}$, is given by:

$$
\begin{aligned}
V_{\text {int }}(x) & =\sqrt{\left|V_{1}^{2}-V_{2}^{2}\right|} \\
& =\sqrt{\left|\frac{1}{2} \frac{\gamma M_{1}}{x_{0}+x}-\frac{1}{2} \frac{\gamma M_{2}}{x_{0}-x}\right|}
\end{aligned}
$$

where $x_{0}$ is positively defined, but $x$, in the term of $M_{1}$, is initially negative, but becomes positive beyond the origin (CM), while, in the term of $M_{2}, x$ is initially positive, but becomes negative below the origin. If $M_{1}=M_{2}, V_{\text {int }}(x=0)=0$. If $x=-x_{0}, V_{\text {int }}(x)$ points upwards and is large near $M_{1}$ (see Fig.3) and for $x=x_{0}, V_{\text {int }}(x)$ points downwards and is large near $M_{2}$. The opposite velocity field within the binary orbit is not at all strange. In many galaxies a central region rotates oppositely to the disk. NGC 7331 is a well-known example in which the central bulb is rotating oppositely to the galactic disk.[18] However, many other examples exist.

In the other regions in the XY plane, the velocity field is strongly affected by continuity and conservation of volume. However, for large $r$ the velocity field tends to the form given by Eq.(7). Along the axis of Y, the velocity of the HQS may increase continuously for decreasing $y$, however the velocity gradient may gradually fall and vanish at $Y=0$. The difficulty to describe the velocity field in such regions however is specific to the binary system. For more symmetric systems, like galaxies, this problem is absent.

\section{The Observed Rotation of Galaxies without the Need of Dark Matter.}

The previous Section III makes a quantitative analysis in terms of the new theory, in which the velocity field of the Higgs Quantum Space (HQS), rules the inertial motion of matter and governs the gravitational dynamics of a binary star system. Increasing the number of stars, moving in the same sense along the same circular orbit round the CM, the oppositely rotating internal and the directly rotating external velocity fields of the HQS become increasingly smoother. Fig. 4 displays a sketch of the collective velocity field, created by a system of eight equal stars, the individual velocity fields of which rotate round axes normal to the figure in the same sense as the orbital velocity of the stars. The figure shows that, due to the orbital velocity and the opposite velocity fields from opposite sides, the opposite internal collective velocity field remains much smaller than the external collective velocity field and that a stagnation point exists at the center and between each pair of stars. The eight stars simply 
move together with the local HQS in their collective velocity field. Precise computation of the velocity field of a galaxy,

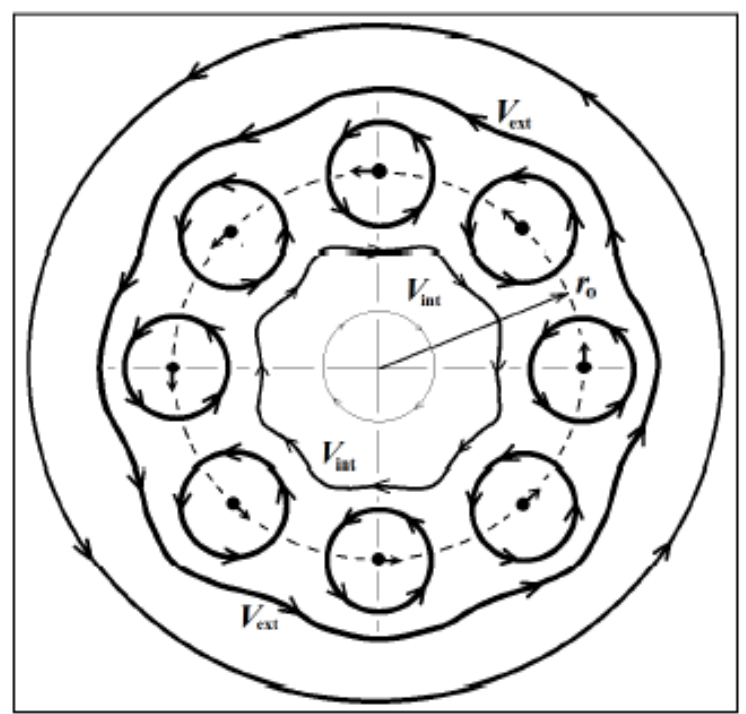

Figure 4. Sketch of the collective velocity field, generated by the polarized velocity fields of eight equal stars, commoving with the HQS along the same circular orbit of radius $r_{0}$ round the CM. The strength of the velocity field is indicated by the broadness of the velocity track. The aim of the figure is showing that, increasing the number of masses in the same orbit loop, the velocity field becomes increasingly smoother. Note that the velocity field of the HQS, inside the orbit, is much weaker and opposite to the orbital motion of the stars and opposite to the considerably stronger external velocity field. Note also that there is a stagnation point at the center and between each pair of stars.

containing hundreds of billions of stars, obviously requires formidable computational means. Here only a qualitative estimate is possible, which however convincingly shows that the HQS-dynamics gravitational mechanism consistently and naturally may lead to the observed non-Keplerian rotation of the galaxies, even with details .

In order to extend the model of the star-loop in Fig.4 closer to the realistic situation of the galactic disk, consider multiple concentric and coplanar circular orbit loops with larger and larger radii and each loop containing a very large number of stars, moving in the same sense round the CM. Fig.5 is a representation of the overall velocity field of the HQS $V_{n}$ and of the estimated effective increase of the velocity of the stars (blue line) through four successive loops in an intermediate region of $r$.

According to Eqs.(12) and (13), each star loop creates a velocity step $\Delta V$, given by Eq.(14), where however $M$ is proportional to the total mass in the loop. This velocity step arises not simply by increasing the velocity of the HQS outward the loop, however also by depressing it inward. While the orbital velocity adds to the symmetric Keplerian velocity field (Eq.(3) of the individual stars outward the loop, it subtracts inward. The orbital velocity makes the velocity field of the stars asymmetric from the viewpoint of the observer in the static non-rotating (XY) reference. This shows that each star loop in the model (each star in the galactic disk) acts in the sense of reducing the Keplerian decrease $\left((1 / r)^{1 / 2}\right.$ of the velocity field, both by lowering the velocity field inward and increasing it outward by the orbital velocity of the loop. Because of this peculiar distortion in the velocity field within the galaxy, such effects are hardly observable from outside, because, as seen in the case of the binary, the velocity fields, created by opposite sides in the orbit or orbit-loop add up in such a way that the effects of the orbital velocity cancels outside the loop. Therefore, depending on the variation of the star density ( $M$ in Eq. (14)) with $r$, the orbital velocity of the stars within the galactic disk and of the velocity of the HQS in the collective velocity field may even increase with $r$, as observed in some galaxies. In terms of the gravitational potential, each successive star loop acts in the sense of lowering the value of the gradient of the collective gravitational potential within the disk and depending on the star density may even invert it.

From the viewpoint of the current gravitational theories, the stars in the galactic disk too attenuate the (inward) gradient of the gravitational potential within the galactic disk. This decrease however is much too small to match the observations. The reason is that, in these theories, the profile of the gravitational potential within the galaxy depends only on the position of the sources and not on their orbital velocity.

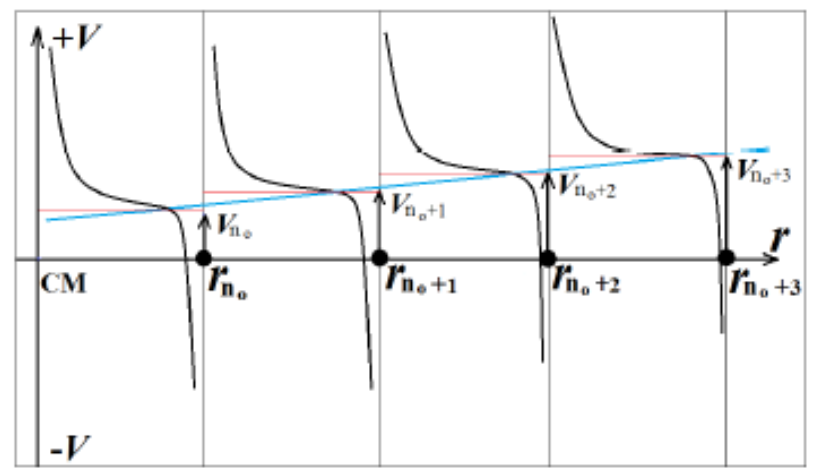

Figure 5. Sketch of the velocity profile of the collective velocity field of the HQS for a system of a large number of loops, along one given $r$ coordinate, through four intermediate star loops, for a case in which the star density $M$ is constant with $r$. The distance from the CM to the loops is indicated by $r_{n}$ $(\mathrm{n}=1,2,3 \ldots)$. The outward distance on from any given star loop $r_{n}$ is $r-r_{n}$, and the separation between successive concentric loops $r_{n+1}-r_{n}$ may be constant. However, in general the mass content within the successive loops may undergo variations with $r$ specific to each galaxy.

In the model of the star loops, it is necessary to consider that the value of the velocity of the HQS decreases with the distance on from each loop $\left(r_{n}\right)$, approximately according to the Keplerian dependence, until the next loop where it increases by another step. This decrease may be approximately given by $C /\left(\left|r-r_{n}\right|\right)^{1 / 2}$, where $C$ is a constant proportional to the square-root of the total mass within the given loop. If the mass density remains constant with $r$, the velocity in the velocity field necessarily increases with $r$, as shown in the sketch of Fig.5. If the star density decreases moderately with $r$, the field velocity eventually may become constant with $r$ and for an even steeper decrease, the velocity eventually may fall with distance.

The star loop model, developed here, is only an approximate qualitative description. It however can convincingly explain the observed gravitational dynamics of galaxies and even predict incredible details, without the need of dark matter. For instance, if the mass density increases very steeply with $r$, the velocity in the velocity field increases very steeply too. This is not a guess. The velocity profile of our Milky Way galaxy in Fig. 1 shows a profound depression and possibly even retrograde rotation close to the galactic center, where the star density becomes low. This depression at the origin is a general feature present in most observed galactic velocity profiles. If the mass density is very low in an extensive region near to the center, the star loop model predicts 
the formation of a central region, where the rotation is in the retrograde sense. In many galaxies, such retrograde rotation is effectively observed. NGC 7331 is an example of a galaxy in which the bulge rotates in the opposite sense to that of the external disk.[18] However, many other examples exist. Beyond the galactic border $r=R$, where the star density begins to fall steeply to zero, the velocity field recovers the Keplerian $(1 / r)^{1 / 2}$ dependence, however on from its value $V=(\gamma M / R)^{1 / 2}$ at the border.

The above analysis evidences that the HQS-dynamic gravitational mechanism provides the physics background that naturally predicts the observed non-Keplerian galactic rotation without the need of dark matter. Hence, from the viewpoint of this new theory, the observed galactic gravitational dynamics is not at all strange. Even the retrograde rotation, observed at the center of many galaxies, is a natural outcome. Clearly, the stars within the galactic disk attenuate the value of the gradient in the original Keplerian velocity field Eq.(3) and, depending on the variation of the star density, may even invert it.

The planets in the solar system constitute a disk round the sun of very low mass density, less than $1 \%$ of the matter of the solar system. They however too must attenuate a little bit the solar Keplerian velocity field until the border of the solar system, where it regains the Keplerian dependence. This has as consequence that the solar gravitational acceleration increases a little bit on going beyond the border of the solar system. This may explain the Pioneer anomaly, which is a very small but well-defined (anomalous) increase in the gravitational acceleration of the Pioneer 10 and Pioneer 11 spacecrafts toward the sun, observed as they moved away from the solar system in opposite directions.[19, 20] To now this anomalous effect never has got and explanation.

Other experimental observations too corroborate the conclusion of the theory of the HQS-dynamics about the nonKeplerian galactic rotation. The null results of the Michelson experiments searching for light anisotropy due to the orbital and cosmic motion of earth demonstrate that the solar system, despite its orbital velocity at about $230 \mathrm{~km} / \mathrm{sec}$ round the galactic center, is closely stationary with respect to the local HQS ruling the propagation of light. However, our solar system cannot be an exception. All the stars within the galactic disk must be closely commoving with the HQS. This will say that the equator of the galactic velocity field of the HQS, creating the galactic gravitational field, coincides with the galactic disk. The stars are of course not constrained to move along these circular orbits by gravitational forces and these circular motions also cannot be explained in terms of spacetime curvature. They simply are carried along circular orbits by the galactic velocity field of the HQS, analogously as the planets are carried round the sun by the solar Keplerian velocity field. These stars are stationary with respect to the local HQS and earth is resting with respect to the local HQS in the solar velocity field, which explains the observed isotropy of light with respect to earth. Moreover, identical clocks commoving with earth and with the solar system or with any other star in the galactic disk, rest with respect to the HQS and run all naturally synchronous, showing all the same universal proper time. However, relative velocities of astronomical bodies, even resting at different places with respect to the local HQS, introduce frequency changes equivalent to usual Doppler effects. The observed isotropy of light with respect to earth shows moreover that the recession between the galaxies and the expansion of the universe are expansion of the HQS itself.[21]

\section{Conclusions}

The Higgs quantum space dynamics gravitational mechanism, has been shown in References [9, 11, 12, 13] to straightforwardly explain the gravitational dynamics on earth and in the solar system. It moreover predicts correctly all the effects of the gravitational fields on the propagation of light and on the rate of clocks. The present work shows that this Higgs quantum space dynamics also naturally reproduces even with incredible details the observed gravitational dynamics of the galaxies, without the need of dark matter. Dark matter is clearly a strange and fictitious ingredient, introduced to keep alive a wrong view about the nature of space and of the gravitational physics.

\section{REFERENCES}

[1] V. Rubin, W. K. Ford, Jr, Astrophysical Journal 159, 379 (1970).

[2] V. Rubin, N. Thonnard, W.K. Ford, Jr, Astrophysical Journal 238, 471 (1980).

[3] Grant Remmen. Journal of Undergraduate Research in Physics, August, pg. 1 (2010).

[4] B. Barbanis and K.H. Prendergast, The Astronomical Journal, 72 72, 215 (1966).

[5] R.R. Hatch, Physics Essays 20, 83 (2007).

[6] R.R. Hatch, GPS Solutions 8, 67 (2004).

[7] R.R. Hatch, Foundations of Physics, 34, 1725 (2004).

[8] Neil Ashby, Private communication, Nov. 5, 2012.

[9] Jacob Schaf, Universal Journal of Physics and Application 9, 141-156, 2015.

[10] H. Bailey, K. Borer, F. Combley, H. Drumm, F. Krienen, Nature 268, 5618 (1977).

[11] Jacob Schaf, Recent Progress in Space Technology, 4, 44-66 (2014).

[12] Jacob Schaf, Journal of Modern Physics, 4, 676-685, (2013).

[13] Jacob Schaf, Journal of Modern Physics 3, 714-749, (2012).

[14] P.W. Anderson, Phys. Rev. 130, 439 (1963).

[15] P. W. Higgs, Physical Review Letters, 13, 508 (1964).

[16] F. Englert and R. Brout, Phys. Rev. Lett. 13, 321, (1964).

[17] J. Schaf, Journal of Modern Physics, 5, 1617-1639, (2014).

[18] F. Prada, C. Gutierrez, R.F. Peletier, C.D. McKeith (14 March 1996) arXiv:astro-ph/9602142. 
[19] S.G. Turyshev, V.T. Toth, âĂIJThe Pioneer AnomalyâĂİ, arXiv:1001.3686v1 19 Aug (2010) (v2).

[20] J.D. Anderson, P.A. Laing, E.L. Lau, A.S. Liu, M.M. Nieto and S.G. Turyshev, Phys. Rev. D, 65, (2002).
[21] J. Schaf, Universal Journal of Physics and Application, "Dark Energy: Accelerated Expansion of the Universe and of the Higgs Quantum Space" (accepted, to come in the Universal Journal of Physics and Application). 